\title{
PROMOÇÃO À SAÚDE COM TRABALHADORES DE SAÚDE MENTAL
}

\section{Health promotion with mental health workers \\ Promoción de la salud de trabajadores sanitarios}

\author{
Patrícia Bitencourt Toscani Greco (iD \\ Universidade Regional Integrada do Alto Uruguai e das Missões - URI - Santiago (RS) - Brasil
}

Alires Morozini Dias

Universidade Regional Integrada do Alto Uruguai e das Missões - URI - Santiago (RS) - Brasil

Camila Milene Soares Bernardi iD

Universidade Regional Integrada do Alto Uruguai e das Missões - URI - Santiago (RS) - Brasil

Danúbia Moraes Legramante iD

Universidade Regional Integrada do Alto Uruguai e das Missões - URI - Santiago (RS) - Brasil

Emanuelli Mancio Ferreira da Luz (iD

Universidade Federal de Santa Maria - UFSM - Santa Maria (RS) - Brasil

Marina Mazzuco de Souza (iD

Universidade Federal de Santa Maria - UFSM - Santa Maria (RS) - Brasil

Juliana Dal Ongaro (iD

Universidade Federal de Santa Maria - UFSM - Santa Maria (RS) - Brasil

\section{RESUMO}

Objetivo: Relatar a experiência de discentes de graduação no desenvolvimento de ações de promoção à saúde com trabalhadores de saúde mental. Síntese dos dados: Trata-se de um relato de experiência realizada por discentes de Enfermagem de uma universidade do Rio Grande do Sul, Brasil, de desenvolvimento de ações de promoção à saúde com trabalhadores de saúde mental. Foram realizados oito encontros, no período de 2015 a 2016, cujos temas destinaram-se à reflexão e discussão do processo de trabalho e qualidade de vida dos profissionais, bem como à realização de atividades para promoção da saúde ocupacional, a partir de espaços de reflexão e atividades de promoção da saúde e prevenção de agravos. Conclusão: As atividades realizadas pelos discentes de graduação junto à população de trabalhadores de saúde mental oportunizaram um olhar diferenciado sobre os aspectos que envolvem a saúde do trabalhador, bem como propiciaram o desenvolvimento de um trabalho em equipe e interdisciplinar entre os cursos de graduação.

Descritores: Enfermagem; Saúde do trabalhador; Promoção da Saúde; Educação em Saúde; Saúde Mental.

\section{ABSTRACT}

Objective: To report the experience of undergraduate students in the development of health promotion actions with mental health workers. Data Synthesis: This experience report was developed by nursing students from a university in Rio Grande do Sul, Brazil, to address the development of health promotion actions with mental health workers. A total of eight meetings were held from 2015 to 2016 and the themes addressed were aimed at the reflection and discussion about the work process, professionals' quality of life, and performance of activities to promote occupational health using spaces for reflection and activities to promote health and prevent diseases. Conclusion: The activities held by undergraduate students with the population of mental health workers favored a differentiated look at the aspects that involve occupational health and allowed the development of interdisciplinary teamwork among undergraduate courses.

Descriptors: Nursing; Occupational Health; Health Promotion; Health Education; Mental Health.

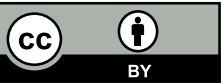




\section{RESUMEN}

Objetivo: Relatar la experiencia de discentes de graduación para el desarrollo de acciones de promoción de la salud de trabajadores de salud mental. Síntesis de los datos: Se trata de un relato de experiencia realizada por discentes de Enfermería de una universidad de Rio Grande do Sul, Brasil, sobre el desarrollo de acciones de promoción de la salud de trabajadores de salud mental. Se realizaron ocho encuentros en el periodo entre 2015 y 2016 cuyos temas se han destinado para la reflexión y discusión del proceso de trabajo y calidad de vida de los profesionales así como la realización de actividades para la promoción de la salud laboral a partir de espacios para reflexión y actividades de promoción de la salud y prevención de agravios. Conclusión: Las actividades realizadas por los discentes de graduación con la población de trabajadores de salud mental ofrecieron una mirada diferenciada de los aspectos que involucran la salud del trabajador así como ha promovido el desarrollo de un trabajo en equipo e interdisciplinar entre los cursos de grado.

Descriptores: Enfermería; Salud Laboral; Promoción de la Salud; Educación en Salud; Salud Mental.

\section{INTRODUÇÃO}

O Ministério da Saúde (MS), por meio da Política Nacional em Saúde Mental, reafirma a importância e a necessidade de planejar a assistência às pessoas com necessidades de tratamento e cuidados específicos em saúde mental ${ }^{(1)}$. Esta política busca promover a integração social e fortalecer a autonomia, o protagonismo e a participação social dos indivíduos que apresentam transtorno mental.

Nota-se que o campo de saúde mental passou por mudanças no modelo assistencial, que perpassa a descentralização dos hospitais para o surgimento dos Centros de Atenção Psicossocial (CAPS). Tais acontecimentos provocaram avanços consideráveis na redefinição de práticas de saúde, com novas concepções sobre o processo saúde-doença( ${ }^{(2)}$. Nesse sentido, entende-se que os profissionais atuantes na saúde mental acabam por ter que realizar mudanças na organização e no processo do trabalho, a fim de adequar-se ao novo modelo de atenção à saúde mental, incluindo o vínculo com usuários e família, a escuta qualificada, entre outras características, o que propicia que esses trabalhadores fiquem expostos ao adoecimento ocupacional ${ }^{(3)}$.

Diante disso, o MS estabeleceu, em 2012, a Política Nacional de Saúde do Trabalhador e da Trabalhadora (PNSTT), que possibilita identificar as atividades realizadas, bem como mapear as situações de risco à saúde, com o intuito de reconhecer as necessidades, demandas e problemas de saúde dos trabalhadores. Ainda, essa política visa promover intervenções, tanto nos ambientes e processos de trabalho como no entorno, tendo em vista a qualidade de vida dos trabalhadores ${ }^{(4)}$.

Destaca-se que a aproximação das relações trabalho-trabalhador tem sido alvo de estudos na área da saúde. Entende-se que o trabalho pode proporcionar prazer, elevar o status, definir a identidade pessoal, impulsionar o crescimento humano, além de atribuir sentido à vida ${ }^{(5)}$. Contudo sabe-se, também, que o ambiente de trabalho ainda é permeado por fatores que interferem negativamente na saúde do trabalhador, o que pode comprometer a sua realização profissional, bem como a sua capacidade para desenvolver as atividades laborais ${ }^{(6)}$.

Dessa forma, é importante ressaltar que os trabalhadores da saúde mental necessitam de estratégias que tenham como objetivo a promoção da saúde. Esta é definida como uma estratégia para buscar a melhoria da qualidade de vida da população. Somado a isso, também objetiva o desenvolvimento de ações para o enfrentamento de problemas, a partir da identificação dos determinantes sociais que interferem na saúde de uma população, para promover a satisfação e bemestar no ambiente laboral ${ }^{(7)}$.

Acredita-se que a promoção da saúde pode prevenir doenças ocupacionais, sendo fundamental na sustentação da capacidade laboral. Com isso, contribui para o fortalecimento de condições favoráveis de trabalho, o bem-estar, a capacidade e qualidade de vida no ambiente laboral, favorecendo a redução do absenteísmo por doenças ocupacionais, da incapacidade (temporária ou permanente), bem como a aposentadoria precoce ${ }^{(8)}$.

A expectativa é de que as ações interdisciplinares (trabalhadores de saúde) de promoção à saúde com os discentes da universidade possam estabelecer um oficio benéfico para os trabalhadores. Dessa forma, ações coletivas possibilitam que a equipe construa estratégias no enfrentamento das demandas e dos estressores inerentes ao processo de trabalho. Sendo assim, possibilitam que se obtenha visibilidade das implicações de suas estratégias em saúde mental, além de trocas de conhecimento e parcerias que potencializam as práticas realizadas neste espaço( ${ }^{(9)}$.

Ao reportar-se para a atuação da Enfermagem como integrante do trabalho interdisciplinar, destacam-se as atribuições direcionadas para o desenvolvimento de atividades de formação técnica, científica e social que são valiosas 
e não visam apenas à capacidade técnica, mas também à reflexão sobre o cuidar. Desse modo, o desenvolvimento de ações relacionadas à saúde do trabalhador contribui para a promoção da saúde e para o incremento na qualidade de vida no trabalho ${ }^{(10)}$.

Nessa perspectiva, a Política Nacional de Promoção da Saúde (PNPS) vem com a proposta de mudanças no trabalho em saúde com vistas a promover a saúde e a qualidade de vida. Considera, ainda, que os setores e instituições são fundamentais para a produção de saúde e do cuidado. Assim, faz-se necessário a consolidação de práticas voltadas para indivíduos e coletividades, em uma perspectiva de trabalho multidisciplinar, integrado e em redes, de forma que considere as necessidades em saúde da população em uma ação articulada entre os diversos atores, em um determinado território(11).

A promoção de saúde pode ser entendida como uma sugestão inovadora de saúde para a população, pois admite a reflexão frente às dificuldades atuais na sociedade e colabora com a importância e incremento de atitudes adequadas para a qualidade de vida ${ }^{(12)}$.

Os índices de adoecimento psíquico em trabalhadores dos serviços de saúde mental, conforme aponta estudo com trabalhadores de CAPS, têm apresentado médio nível de estresse ${ }^{(3)}$ e índices de moderados a críticos de desgaste profissional ${ }^{(5)}$. Ademais, estudo desenvolvido no Sul do país demostra que $20,3 \%$ dos trabalhadores da saúde pública apresentam algum tipo de transtorno mental comum e 11,6\% já, em algum momento, apresentaram ideação suicida(13). Tendo em vista esses resultados, há a necessidade de se vislumbrar oportunidade de produção de saúde e bem-estar no trabalho das equipes de saúde mental.

Assim, a justificativa para a realização da presente experiência partiu do pensamento de que ações educativas se constituem como ferramentas eficazes para disseminar o aprendizado e a autonomia para a promoção da saúde de trabalhadores de saúde mental. Desse modo, a relevância da experiência está em possibilitar aos graduandos de uma universidade comunitária a reflexão e a utilização de ações de educação e promoção da saúde com o intuito de proporcionar e/ ou ampliar o bem-estar e a qualidade de vida dos trabalhadores em saúde mental.

Diante do exposto, o presente artigo tem como objetivo relatar a experiência de discentes de graduação no desenvolvimento de ações de promoção à saúde com trabalhadores de saúde mental.

\section{SÍNTESE DOS DADOS}

Trata-se de um relato de experiência acerca das ações de promoção à saúde com trabalhadores de saúde mental desenvolvidas por discentes de Enfermagem a partir do projeto de extensão intitulado: "Promoção da saúde: um espaço interdisciplinar para os trabalhadores dos Centros de Atenção Psicossocial do município de Santiago", do curso de graduação em Enfermagem da Universidade Regional Integrada do Alto Uruguai e das Missões (URI), campus Santiago, Rio Grande do Sul (RS), Brasil(14). O projeto de extensão está registrado no Sistema de Projetos da URI (SPURI) e no Núcleo de Pesquisa e Extensão (NUPEX) e contou com financiamento de dois editais institucionais: 04/2015 e 06/2016. As ações propostas foram realizadas no período de agosto de 2015 a julho de 2016, nos Centros de Atenção Psicossocial (CAPS) do referido município.

No CAPS, o atendimento é direcionado para atender aos indivíduos com transtornos mentais, com acompanhamento clínico e reinserção social(1). O público-alvo das atividades constituiu-se de uma equipe multiprofissional, ocorrendo integração, vínculo e relacionamento empático entre acadêmicos e os trabalhadores.

No total, realizaram-se oito encontros, cada um com duração aproximada de uma hora. A divulgação do projeto entre os trabalhadores, bem como a organização do espaço para realização da atividade, era de responsabilidade dos discentes envolvidos no projeto de extensão.

As ações de promoção da saúde foram planejadas entre docente e discentes, tendo em vista a realidade do serviço, conforme as sugestões elencadas pelos trabalhadores e em acordo com a Política Nacional de Promoção da Saúde ${ }^{(11)}$. Além disso, o planejamento se dava a partir das sugestões de atividades, bem como das necessidades elencadas pelos trabalhadores.

As ações desenvolvidas nos encontros proporcionaram um espaço de reflexão e discussão sobre o processo de trabalho, a qualidade de vida no ambiente laboral, o reconhecimento, o engajamento e a valorização profissional. Também se contemplou atividades para prevenção de agravos físicos à saúde do trabalhador, tais como: ginástica laboral, circuito funcional, slackline, técnicas de massagem e exercícios físicos e fisioterápicos.

O primeiro encontro foi destinado à sensibilização e à apresentação do projeto de extensão aos trabalhadores. Após, foi proposta uma dinâmica em grupo na qual os trabalhadores demonstrassem, por meio de desenhos e/ ou da escrita, a sua história de vida, contemplando fatos internos e externos ao ambiente laboral, proporcionando 
reflexão sobre o processo de trabalho. Em seguida, cada participante apresentou sua construção ao grande grupo, e então eram coladas em um cartaz coletivo. A partir dessa organização coletiva, construiu-se a história de todos os trabalhadores que atuavam no local, e possibilitou perceber o quanto o trabalho e a vida pessoal estão imbricados. Nesse sentido, os trabalhadores discutiram a necessidade de união e apoio mútuo no espaço de trabalho.

No segundo momento, as atividades tiveram como foco a prevenção de agravos físicos à saúde do trabalhador. Nessa perspectiva, proporcionou-se um espaço para realizar atividade física e movimentar o corpo por meio da ginástica laboral e da dança rítmica com auxílio de um educador físico. Essas atividades propiciaram a interação e a sincronia da equipe, bem como a descontração e a leveza do ambiente. A prática de atividade física no ambiente laboral auxilia na promoção de saúde e na qualidade de vida dos trabalhadores. Além disso, possibilita prevenir doenças que o trabalho repetitivo e monótono pode acarretar, aumentando a disposição do trabalhador e promovendo maior integração no local de trabalho(15).

A terceira atividade foi organizada pelos trabalhadores do CAPS, os quais convidaram um profissional médico para realizar uma fala acerca da motivação e da qualidade de vida laboral. Ressalta-se que as temáticas contemplaram as expectativas dos participantes, o que oportunizou reflexões provenientes do desempenho no trabalho, de modo que se mostraram participativos, interessados e satisfeitos com as ações desenvolvidas. Nesse sentido, a satisfação pessoal e do trabalho, como também a motivação, estão atreladas à qualidade de vida laboral, o que proporciona aumento na produtividade ${ }^{(16)}$.

No quarto encontro, realizaram-se atividades por meio de técnicas de massagem com o auxílio de bolinhas do tipo cravo, as quais os trabalhadores deslizavam sobre seus segmentos corporais individualmente ou em duplas, a fim de proporcionar o relaxamento corporal. Os trabalhadores mostraram-se muito animados e descontraídos com a atividade, perceptível pelo tempo de duração das massagens, as quais ultrapassaram os 60 minutos de atividade propostos pelo projeto, corroborando um estudo no qual massagem no ambiente laboral constituiu-se como uma importante ferramenta preventiva, auxiliando na diminuição de tensão, fadiga, dores musculoesqueléticas e na melhora do humor dos trabalhadores ${ }^{(17)}$.

No quinto e último momento do ano de 2015, ocorreu outra dinâmica em grupo com a intenção de escolher um presidente fictício para o CAPS do município. Para isso, sucedeu-se um sorteio com o nome de cada trabalhador, com a finalidade de cada participante colocar as virtudes que o colega sorteado possuía e, assim, fazer a propaganda dele em forma de recortes de revista. Logo após, todos entraram em um consenso de qual participante representaria melhor o cargo de presidente. Assim, esse encontro possibilitou a valorização dos trabalhadores e reflexões sobre suas contribuições na dimensão multidisciplinar, visto que cada trabalhador procurou demonstrar as qualidades e habilidades do seu colega no ambiente laboral.

O sexto encontro, realizado no ano de 2016, ocorreu com o reencontro do docente e dos discentes com os trabalhadores do CAPS, retomando as ações propostas pelo projeto. Para esse encontro, convidaram discentes do curso de Educação Física, que propuseram atividades físicas por meio de exercícios funcionais com faixas elásticas de diferentes intensidades. A integração dos discentes de ambos os cursos se estendeu ao encontro seguinte.

No sétimo encontro, foi desenvolvida a atividade denominada slackline e um pequeno circuito funcional, que utilizou atividades que contemplavam pular corda, agachar, correr e realizar pulos laterais. O desenvolvimento dessas ações foi importante no ambiente laboral, uma vez que possibilitou a distração e o envolvimento dos trabalhadores e discentes de ambos os cursos. Salienta-se que atividades como estas contribuem para a promoção da qualidade de vida entre trabalhadores da saúde mental ${ }^{(15)}$.

Propôs-se, no oitavo e último encontro, outra dinâmica grupal, abordando o reconhecimento, o engajamento e a valorização profissional. Entregou-se a um dos participantes uma caixa decorada, solicitando-se, em seguida, que fosse entregue sucessivamente a uma pessoa, conforme os diferentes adjetivos que surgiam, de modo que chegasse a todos os participantes. Por fim, pediu-se que a caixa fosse entregue à uma pessoa generosa e, então, o participante contemplado com tal adjetivo abriu o presente para dividir com os demais. Dessa forma, possibilitou que todos os trabalhadores trocassem elogios, qualidades e, assim, pudessem se sentir reconhecidos e valorizados dentro da equipe.

Os encontros possibilitaram discutir sobre valorização e reconhecimento entre os pares, integração e trabalho em equipe, além de proporcionar momentos de atividade física. Dessa forma, forneceram ao trabalhador um espaço de descontração, movimentação e reflexão sobre seus benefícios e a possibilidade de incorporá-la em seu cotidiano. Nesse contexto, um estudo com trabalhadores de saúde mental sugere que sejam ofertados aos trabalhadores programas de aprimoramento. Além disso, também ressalta como gerador de prazer no trabalho espaços que estão permeados por reconhecimento, liberdade e horizontalidade das relações ${ }^{(1)}$. 
Observou-se que as ações propostas pelo presente projeto fortaleceram as relações entre os trabalhadores da equipe e favoreceram a relação entre ensino e serviço. Nessa perspectiva, a prática vem ao encontro de estudo que ressalta como características de qualidade de vida no trabalho: ter condições de trabalho favoráveis; ambiente harmônico; ser valorizado e ouvido; ser respeitado; gostar do que faz; ter oportunidades de desenvolvimento; ter reconhecimento; bom relacionamento com os colegas, entre outros ${ }^{(16)}$.

Ademais, destaca-se a importância da articulação das instituições de ensino e serviços de saúde a fim de fomentar uma formação pautada na realidade do sistema de saúde e com vistas a torna-se parceira na transformação destes, por meio de ações de promoção da saúde, bem como de prevenção de agravos ${ }^{(11)}$.

A avaliação dos discentes sobre o desenvolvimento de ações interdisciplinares de promoção à saúde foi positiva. Observou-se a integração entre os acadêmicos e a equipe, o que possibilitou a vivência dos discentes para além das ações com os trabalhadores do CAPS. Desse modo, a experiência oportunizou aos discentes vivenciarem situações do cotidiano de um serviço de saúde mental, bem como observarem atuação dos trabalhadores de saúde nas atividades de promoção da saúde.

O desenvolvimento de ações interdisciplinares de promoção à saúde de trabalhadores do âmbito da saúde mental justifica-se pelo alto risco de adoecimento físico e psíquico aos quais os trabalhadores encontram-se expostos ${ }^{(3,5)}$. Deve-se atentar para esses trabalhadores, pois além dos riscos ocupacionais comuns, como ruídos, bactérias, adoção de postura corporal inadequada, estresse, entre outros fatores que as instituições de saúde acarretam, no âmbito da saúde mental, a exposição torna-se maior, visto que são locais permeados por tensão emocional em virtude das situações vivenciadas e da imprevisibilidade dos pacientes assistidos ${ }^{(18)}$.

Estudos evidenciam uma alta taxa de transtornos mentais associados à sobrecarga psíquica (23,7\% e 20,3\%) ${ }^{(19,13)} \mathrm{em}$ profissionais da saúde, o que, por sua vez, acarreta o afastamento de suas atividades laborais. Trabalhadores da saúde com alta exigência psicológica e baixo controle das demandas laborais têm comumente sido identificados com transtornos mentais comuns ${ }^{(13,20)}$.

Nesse intuito, os riscos à saúde dos profissionais que prestam assistência em saúde mental merecem a atenção dos pesquisadores e dos gestores da saúde pública, em especial daqueles responsáveis por viabilizar serviços humanizados $^{(3)}$. Portanto, oportunizar um espaço com suporte para fala dos trabalhadores talvez possa ser uma maneira de reflexão sobre o processo de trabalho, bem como para realizar atividades destinadas para prevenção de agravos físicos e psíquicos.

É importante ressaltar que é necessário trazer à tona a discussão sobre a saúde do trabalhador já na graduação, a fim de favorecer a formação de profissionais que refletem sobre sua prática, bem como sobre as repercussões desta em sua saúde. Acredita-se que atividades extramuros da universidade, promovidas por meio de projetos de extensão, fortalecem a formação do discente enquanto profissional da saúde, bem como fomentam ações de educação em saúde e facilitam o acesso à informação de trabalhadores.

Diante do exposto, entende-se que o profissional de saúde precisa de qualidade de vida para promover uma assistência à saúde adequada e de qualidade. Os trabalhadores podem deparar-se com diversos problemas no cotidiano, os quais comprometem as funções e atribuições diárias no serviço e que, por vezes, são capazes de afetar a qualidade de vida dos mesmos. Entende-se que a promoção da saúde e do bem-estar do trabalhador favorece para um ambiente laboral mais produtivo e menos prejudicial( ${ }^{(21)}$.

Os encontros planejados pelos estudantes utilizaram metodologias diferenciadas e dinâmicas em grupo. Essas atividades fortaleceram as relações no trabalho, bem como proporcionaram momentos de integração e bem-estar aos trabalhadores. Para os estudantes, planejar ações de promoção à saúde de trabalhadores possibilitou refletir sobre a prática em equipe no trabalho em saúde. Destaca-se que os estudantes necessitam ter ações pautadas em propostas metodológicas que permitam seu crescimento crítico, reflexivo e intelectual para, assim, articularem estratégicas coletivas ${ }^{(10)}$.

Ressalta-se que a universidade tem a responsabilidade e o comprometimento de oportunizar esse espaço de suporte que viabilize a discussão e construção de conhecimento. Dessa forma, a extensão pode ser a via pela qual a universidade divulga seus conhecimentos, tornando-se importante meio de integração entre universidade e comunidade $^{(22)}$. A extensão universitária possibilita aliar teoria e prática na busca por compartilhar conhecimento e vivenciar a realidade laboral.

As ações de promoção à saúde poderão auxiliar os trabalhadores a desenvolver práticas de autocuidado e a enfrentar as demandas e os estressores inerentes ao processo de trabalho. Para os trabalhadores dos CAPS, destaca-se que oportunizar melhorias na qualidade de vida no trabalho pode representar uma redução do índice de absenteísmo por doenças ocupacionais, bem como melhor qualidade de vida. 
As atividades físicas promovidas nos encontros ocorridos no projeto de extensão favorecem a perspectiva de prevenção de doenças que o trabalho repetitivo e monótono pode acarretar. Reconhece-se que a promoção em saúde, por meio de atividades de ginástica laboral, pode influenciar positivamente a qualidade de vida dos trabalhadores ${ }^{(23)}$.

O trabalhador em saúde exerce um esforço físico e cognitivo em suas funções, o que necessita uma extrema atenção, equilíbrio e cuidado ao cumpri-las. Além disso, estão inseridos em um meio com alta probabilidade de desencadear o estresse e, consequentemente, sobrecargas físicas e psicológicas, que provocam a diminuição da autoestima e do relacionamento interpessoal. Considerando-se essas questões, observa-se que a motivação auxilia nesse processo de melhora na qualidade de vida no trabalho. Ao proporcionar um conjunto de ações aos trabalhadores em seu ambiente laboral, oportunizam-se a satisfação e harmonia em suas relações na sociedade ${ }^{(24)}$.

A qualidade de vida no trabalho está ligada ao desenvolvimento humano e a aspectos sociais e econômicos, assim como ao estado de saúde do indivíduo, à satisfação no trabalho e às relações familiares. Quanto à saúde do trabalhador, faz-se necessária a implementação de intervenções de promoção e proteção da saúde que reduzam os riscos presentes no ambiente laboral. Nota-se que proporcionar aos trabalhadores oportunidades em projetos novos e programas de educação continuada minimiza o processo saúde-doença, possibilita a qualificação profissional e, posteriormente, sua valorização(8).

Acredita-se que os encontros de extensão se configuraram como uma estratégia para a promoção da saúde do trabalhador do CAPS, pois possibilitaram subsídios para a melhora da qualidade de vida deles por meio de atividades físicas, lúdicas, de lazer e de intervenção. Esses tipos de ações de promoção da saúde propiciam trocas de experiências, e de conhecimentos acerca da temática envolvida, estimulando a autonomia e o pensamento crítico dos envolvidos ${ }^{(25)}$. Reforça-se que os participantes poderão ser multiplicadores entre seus pares, em seus postos de trabalho, uma vez que estratégias de enfrentamento podem ser apreendidas e ampliadas, bem como as demais ações de promoção à saúde ocupacional.

As ações do projeto de extensão, além de contribuírem para a saúde e bem-estar dos trabalhadores, possibilitaram um ambiente de trabalho mais leve e um espaço para integração, reflexão, autocuidado e cuidado entre os colegas. Corroborando com o descrito, um estudo destaca que ações de promoção saúde proporcionam melhores condições de vida e saúde, tanto nos serviços quanto na vida do trabalhador, sendo uma forma eficaz de produzir saúde no âmbito laboral voltado para diferentes públicos: trabalhadores multiprofissionais de saúde, do ensino, de empresas, entre outros ${ }^{(26)}$.

Acredita-se que as atividades desenvolvidas no projeto de extensão corroboram os objetivos da PNPS, tendo em vista que algumas das ações desenvolvidas estão interligadas a ela, como: o desenvolvimento de espaços de produção social e de um ambiente saudável, que favorece para o desenvolvimento humano e o bem-viver; a promoção do empoderamento, da capacidade de tomada de decisão da autonomia no processo de trabalho; e também a promoção de ações de educação com vistas à promoção da saúde para trabalhadores, de acordo com os princípios e os valores expressos na PNPS ${ }^{(11)}$.

Este estudo possibilitou um impacto positivo, a partir do momento em que o projeto de extensão, após dois anos, tornou-se sistemático nos serviços públicos do município. Isto contribui significativamente para PNPS, visto que as ações foram desenvolvidas visando ao indivíduo e à coletividade, em uma perspectiva de trabalho multidisciplinar, integrado, no intuito de considerar as necessidades em saúde da população, com ações articuladas entre os diversos atores. Além disso, as ações contemplam as diretrizes da PNPS, principalmente no que diz respeito a estimular o desenvolvimento de projetos e pesquisas, e de produção e difusão de conhecimentos e de estratégias inovadoras no âmbito das ações de promoção da saúde ${ }^{(11)}$.

Entende-se que, a partir da experiência relatada, a qual demonstra a efetivação da PNPS, seja possível replicar essas ações de promoção da saúde com os trabalhadores de diversos serviços de saúde, visando à qualidade de vida no trabalho, bem como a articulação teoria e prática sobre saúde do trabalhador por parte dos discentes. No que tange à formação, por sua vez, possibilitará futuros profissionais mais sensíveis e preparados para refletir sobre os processos organizacionais e as repercussões para o trabalhador e para a assistência à saúde. Como limitação encontrada neste estudo, houve a dificuldade de adesão de profissionais, talvez por resistência em discutir sobre a organização do trabalho.

Ainda, destaca-se a necessidade de se utilizar estratégias de promoção de saúde com trabalhadores atuantes nos serviços de saúde pública, independente da complexidade do cuidado envolvido. Dessa forma, articular a PNPS e a PNSTT poderá refletir positivamente na ampliação do olhar dos trabalhadores da saúde no que se refere à produção de saúde e bem-estar dos usuários e famílias. 


\section{CONCLUSÃO}

A participação dos discentes de graduação em Enfermagem nas ações de promoção à saúde com a população de trabalhadores de saúde mental oportunizou um olhar diferenciado sobre os aspectos que envolvem a saúde do trabalhador, bem como oportunizou desenvolver atividades de trabalho em equipe e a interdisciplinaridade entre os cursos de graduação.

Nessa perspectiva, as ações educativas possibilitaram a troca de saberes entre docentes, discentes e trabalhadores, promovendo a construção e multiplicação de conhecimento acerca das temáticas em pauta.

Destaca-se a importância de valorizar ações que promovam a saúde e o bemestar dos trabalhadores, para que possam se sentir reconhecidos, bem como engajados, no cuidado em saúde mental e desenvolvimento profissional. A interdisciplinaridade promoveu o fortalecimento deste estudo e das ações desenvolvidas, uma vez que permitiu ampliar o olhar sobre a saúde do trabalhador.

\section{FONTES DE FINANCIAMENTO}

Sistema de Projetos da URI (SPURI) e o Núcleo de Pesquisa e Extensão (NUPEX).

\section{CONTRIBUIÇÕES}

Patrícia Bitencourt Toscani Greco e Danúbia Moraes Legramante contribuíram com a elaboração e delineamento do estudo; a aquisição, análise e interpretação de dados; e a redação e/ou revisão do manuscrito. Alires Morozini Dias, Camila Milene Soares Bernardi, Emanuelli Mancio Ferreira da Luz, Marina Mazzuco de Souza e Juliana Dal Ongaro contribuíram com a aquisição, análise e interpretação de dados, e a redação e/ou revisão do manuscrito.

\section{CONFLITOS DE INTERESSE}

Os autores declaram a inexistência de conflitos de interesses, tanto na execução das ações do programa de extensão como na descrição deste relato de experiência.

\section{REFERENNCIAS}

1. Ministério da Saúde (BR), Coordenação Nacional de Saúde Mental. Política nacional de saúde mental, álcool e outras drogas [Internet]. Brasília: Ministério da Saúde; 2017 [acesso em 2019 Mar 23]. Disponível em: http://portalms.saude.gov.br/politica-nacional-de-saude-mental-alcool-e-outras-drogas

2. Vasconcelos MGF, Jorge MSB, Catrib AMF, Bezerra IC, Franco TB. Projeto terapêutico em Saúde Mental: práticas e processos nas dimensões constituintes da atenção psicossocial. Interface (Botucatu) [Internet]. 2016 [acesso em 2019 Mar 23];20(57):313-23. doi: http://dx.doi.org/10.1590/1807-57622015.0231

3. Bellenzani R, Paro DM, Oliveira MC. Trabalho em saúde mental e estresse na equipe: questões para a Política Nacional de Humanização/SUS. Rev Psicol Saúde [Internet]. 2016 [acesso em 2019 Mar 23];8(1):3243. doi: http://dx.doi.org/10.20435/2177093X2016105

4. Ministério da Saúde (BR). Portaria $n^{\circ} 1.823$, de 23 de agosto de 2012. Institui a Política Nacional de Saúde do Trabalhador e da Trabalhadora [Internet]. Ministério da Saúde: Brasília; 2012 [acesso em 2019 Mar 23]. Disponível em: http://bvsms.saude.gov.br/bvs/saudelegis/gm/2012/prt1823_23_08_2012.html

5. Gonçalves AM, Vilela SC, Terra FS, Nogueira DA. Atitudes e o prazer/sofrimento no trabalho em saúde mental. Rev Bras Enferm [Internet]. 2016 [acesso em 2019 Set 29];69(2):245-53. doi: http://dx.doi. org/10.1590/0034-7167.2016690209i

6. Andolhe R, Barbosa RL, Oliveira EM, Costa ALS, Padilha KG. Estresse, coping e burnout da Equipe de Enfermagem de Unidades de Terapia Intensiva: fatores associados. Rev Esc Enferm USP [Internet]. 2015 [acesso em 2019 Mar 24];49(Esp):58-64. doi: 10.1590/S0080-623420150000700009

7. Ministério da Saúde (BR), Secretaria-Executiva, Secretaria de Vigilância em Saúde. Glossário temático: promoção da saúde [Internet]. Ministério da Saúde: Brasília; 2013 [acesso em 2019 Mar 24]. Disponível em: http://bvsms.saude.gov.br/bvs/publicacoes/glossario_tematico_promocao_saude.pdf 
8. Andrade KO, Andrade PO, Leite LF. Qualidade de vida dos trabalhadores da área de saúde: revisão de literatura. Rev Cient ITPAC [Internet]. 2015 [acesso em 2019 Mar 24];8(1):1-5. Disponível em: https://assets. itpac.br/arquivos/Revista/76/Artigo_1.pdf

9. Miranda GU, Santos CF, Cunha JG, Martins TCC, Ribeiro RO, Dias LA. Programa "Cada doido com sua mania": promoção de saúde mental e formação interdisciplinar em instituição aberta para crianças, adolescentes e adultos. Rev Extensão Guará [Internet]. 2015 [acesso em 2019 Mar 24];(3):93-100. doi: https://doi.org/10.30712/guara.v3i3.9840

10. Faria MGA, Gallasch CH, Martins ALX. Inserção de graduandos de enfermagem em serviços de saúde do trabalhador: relato de experiência. Cogitare Enferm [Internet]. 2016 [acesso em 2019 Mar 27];21(4):01-07. doi: http://dx.doi.org/10.5380/ce.v21i4.45245

11. Ministério da Saúde (BR), Secretaria de Vigilância em Saúde, Secretaria de Atenção à Saúde. Política Nacional de Promoção da Saúde: PNPS: Anexo I da Portaria de Consolidação $n^{\circ} 2$, de 28 de setembro de 2017, que consolida as normas sobre as políticas nacionais de saúde do SUS [Internet]. Brasília: Ministério da Saúde; 2018 [acesso em 2019 Set 29]. Disponível em: http://bvsms.saude.gov.br/bvs/publicacoes/politica_ nacional_promocao_saude.pdf

12. Westphal MF. Promoção da saúde e qualidade de vida. In: Fernandez JCA, Mendes R, organizadores. Promoção da saúde e gestão local. São Paulo: Huvitec-Cepedoc; 2007. p. 17-21.

13. Faria NMX, Klosinski RFS, Rustick G, Oliveira LM. Saúde mental dos trabalhadores da saúde pública em Bento Gonçalves, no Rio Grande do Sul. Rev Bras Med Trab [Internet]. 2018 [acesso em 2019 Apr 04];16(2):145-57. doi: 10.5327/Z1679443520180196.

14. Universidade Regional Integrada do Alto Uruguai e das Missões. Projeto político pedagógico do curso de enfermagem 2016 [Internet]. 2016 [acesso em 2019 Out 08]. Disponível em: http://www.reitoria.br/ soap/ projetospedagogicos.php

15. Duarte MS, Gonçalves NR, Ferreira GNT, Cunha RG. O impacto de um programa de ginástica laboral mensurado através do questionário nórdico de sintomas. Rev e-Scientia [Internet]. 2017 [acesso em 2019 Set 29];10(1):1-12. Disponível em: https://revistas.unibh.br/dcbas/article/view/2007/pdf

16. Ferreira CAA, Vasconcelos FCW, Goulart IB, Ituassu CT. A qualidade de vida no trabalho: uma visão crítica dos trabalhadores da saúde mental. Rev Eletrônica Fafit/Facic [Internet]. 2015 [acesso em 2019 Set 29];6(2):13-30. Disponível em: http://www.fafit.com.br/revista/index.php/fafit/article/view/134

17. Durães GM, Lafetá JC, Maia MFM, Silveira MG, Gonçalves JM. Massagem na saúde laboral: Influência no comportamento motor e estresse ocupacional. Educ Fís Deporte [Internet]. 2015 [acesso em 2019 Set 29];34(2):555-74. doi: 10.17533/udea.efyd.v34n2a11

18. Fernandes MA, Marziale MHP. Riscos ocupacionais e adoecimento de trabalhadores em saúde mental. Acta Paul Enferm [Internet]. 2014 [acesso em 2019 Apr 02];27(6):539-47. doi: http://dx.doi.org/10.1590/19820194201400088

19. Silva-Junior JS, Fischer FD. Afastamento do trabalho por transtornos mentais e estressores psicossociais ocupacionais. Rev Bras Epidemiol [Internet]. 2015 [acesso em 2019 Apr 02];18(4):735-44. doi: 10.1590/19805497201500040005

20. Rodrigues EP, Rodrigues US, Oliveira LMM, Laudano RCS, Nascimento CL Sobrinho. Prevalência de transtornos mentais comuns em trabalhadores de enfermagem em um hospital da Bahia. Rev Bras Enferm [Internet]. 2014 [acesso em 2019 Apr 05];67(2):296-301. doi: http://dx.doi.org/10.5935/0034-7167.20140040

21. Lobo LMGA, Oliveira FBM. Qualidade de vida dos profissionais de enfermagem: uma revisão integrativa de literatura. Rev ReOnFacema [Internet]. 2015 [acesso em 2019 Apr 05];1(1):86-91. Disponível em: http://www. facema.edu.br/ojs/index.php/ReOnFacema/article/view/23/19

22. Oliveira FLB, Almeida JJ Jr. Extensão universitária: contribuições na formação de discentes de Enfermagem. Rev Bras Pesq Saúde [Internet]. 2015 [acesso em 2019 Apr 08];17(1):19-24. Disponível em: http://www. portaldepublicacoes.ufes.br/RBPS/article/viewFile/12445/8655

23. Silva RSB, Martins CO, Rosentiel L, Ferreira CNF, Silva AS. Influência de informações de saúde no estilo 
de vida de participantes de ginástica laboral. Rev Bras Promoç Saúde [Internet]. 2014 [acesso em 2019 Apr 10];27(3):1-7. Available from: https://periodicos.unifor.br/RBPS/article/view/2708/pdf

24. Sganzerla CM, Martini C, Tasca FJ, Fuzinatto L. Qualidade de vida do trabalhador: estresse e motivação no cotidiano [Internet]. In: Anais do I Seminário macrorregional de Saúde Coletiva; 2017 Out. 26-27; São Miguel do Oeste. São Miguel do Oeste: Unoesc; 2017 [acesso em 2019 Apr 10]. Disponível em: http://editora. unoesc.edu.br/index.php/SMSC/article/viewFile/16642/9033\#page $=65$

25. Munhoz OL, Ramos TK, Moro B, Timm MS, Venturini L, Cremonese L, et al. Oficina bingo da saúde: uma experiência de educação em saúde com grupos de idosos. REME Rev Min Enferm [Internet]. 2016 [acesso em 2019 Apr 12];20(e968):1-6. doi: 10.5935/1415-2762.20160038

26. Carvalho FFB, Cohen SC, Akerman M. Refletindo sobre o instituído na Promoção da Saúde para problematizar 'dogmas'. Saúde Debate [Internet]. 2017 [acesso em 2019 Oct 05];41(spe3):265-76. doi: http:// dx.doi.org/10.1590/0103-11042017s320 11042017s32042017s32011042017s320

\section{Endereço para correspondência:}

Patrícia Bitencourt Toscani Greco

Universidade Regional Integrada do Alto Uruguai e das Missões - URI

Av. Batista Bonoto Sobrinho

Bairro: São Vicente

CEP: 97700-000 - Santiago - RS - Brasil

E-mail: pbtoscani@hotmail.com

Como citar: Greco PBT, Dias AM, Bernardi CMS, Legramante DM, Luz EMF, Souza MM, et al. Promoção à saúde com trabalhadores de saúde mental. Rev Bras Promoç Saúde. 2019;32:9669. 